\title{
CONSTRUCCIONES DISCURSIVAS SOBRE EL PASADO EN EL ARCHIVO DE HISTORIA ORAL DE LA UNIVERSIDAD NACIONAL DEL LITORAL
}

\author{
CONSTRUÇÕES DISCURSIVAS SOBRE O PASSADO NO ARQUIVO DE \\ HISTÓRIA ORAL DA UNIVERSIDADE NACIONAL DO LITORAL
}

Fabiana Alonso

Universidad Nacional del Litoral - UNL fabianaalonso11@hotmail.com

Eliana Bertero Universidad Nacional del Litoral - UNL elibertero@gmail.com

\begin{abstract}
Resumen
La producción testimonial es una práctica que viene siendo desarrollada desde el siglo XX en la investigación social. En las últimas décadas, distintos planteos teórico-metodológicos han abordado las fuentes orales como interpretaciones acerca del pasado y se han desarrollado indagaciones tendientes a esclarecer los procesos de creación de significados, el peso del presente en el acto de rememoración y la configuración del testimonio, entre otras cuestiones que hacen a su especificidad. La organización de un archivo de historia oral obedece a la preocupación por la preservación de fuentes orales producidas en virtud del diálogo entre un investigador y un testigo, seleccionado por su participación en determinado proceso histórico. La archivación de testimonios orales requiere fijar criterios prácticos para facilitar su conservación y accesibilidad en el marco de un campo que ha adquirido una notable expansión. El presente artículo da cuenta de la organización de un archivo oral en la Universidad Nacional del Litoral (Santa Fe, Argentina), actividad emprendida desde mediados de 2010. Se explicitan los criterios para la formación de colecciones de entrevistas, el formato de grabación y las tareas de sistematización que posibilitan la consulta de las fuentes orales por investigadores de distintas disciplinas de las ciencias sociales.
\end{abstract}

Palabras clave: Testimonio. Pasado. Presente. Archivo. Investigación. 


\section{Resumo}

A produção testemunhal é uma prática desenvolvida desde o século XX na pesquisa social. Nas últimas décadas, diferentes propostas teórico-metodológicas abordaram fontes orais como interpretações sobre o passado e pesquisas foram desenvolvidas para esclarecer os processos de criação de significados, o peso do presente no ato de recordar e a configuração do testemunho, entre outras questões que compõem sua especificidade. A organização de um arquivo de história oral obedece à preocupação com a preservação de fontes orais produzidas em virtude do diálogo entre um pesquisador e uma testemunha, selecionada por sua participação em determinado processo histórico. $\mathrm{O}$ arquivamento de testemunhos orais requer a definição de critérios práticos para facilitar a sua conservação e acessibilidade no âmbito de um campo que adquiriu uma notável expansão. Este artigo dá conta da organização de um arquivo oral na Universidad Nacional del Litoral (Santa Fe, Argentina), uma atividade realizada desde meados de 2010. Os critérios para a formação de coleções de entrevistas são explicitados, igualmente o formato de gravação e as tarefas de sistematização que permitem consultar fontes orais por pesquisadores de diferentes disciplinas das ciências sociais.

Palavras-chave: Testemunho. Passado. Presente. Arquivo. Pesquisa.

\section{1- Introducción}

La Universidad Nacional del Litoral (UNL) se creó en 1919 luego de un proceso iniciado a comienzos de la década, a raíz de los reclamos por la nacionalización de la entonces Universidad de Santa Fe. La movilización social y política, que tuvo como principal referente al movimiento estudiantil santafesino, se profundizó con el reformismo universitario de $1918^{\mathrm{i}}$. En ese contexto, la creación de la UNL - con facultades en las ciudades de Santa Fe, Rosario, Paraná y Corrientes- sintetizó las demandas de un conjunto de universitarios, políticos y miembros de la sociedad civil, quienes impulsaron un proyecto modernizador de carácter reformista y regional. Algunos fueron figuras influyentes en el gobierno de la universidad y desde allí proyectaron y organizaron una institución que construyó tempranamente una tradición reformista. Así, en los primeros años de ordenamiento institucional y administrativo fue encarada la organización de los archivos universitarios.

En 1927, el rector Pedro Martínez visualizó la importancia de organizar un archivo en el que pudiera resguardarse la historia documentada de la universidad desde su creación. En la memoria de su gestión sostenía que el valor de la documentación sería apreciado en el futuro 
cuando quisieran tenerse referencias y antecedentes precisos del pasado de la UNL ${ }^{\mathrm{ii}}$. Sin embargo, y a pesar de este impulso inicial, en su trayectoria posterior la UNL no estuvo ajena a la combinación de diversas contingencias como la discontinuidad institucional, los problemas presupuestarios, la ausencia de políticas archivísticas, la negligencia burocrática o las intervenciones militares que ejercieron una verdadera devastación sobre todo el material documental que consideraron "subversivo", situación compartida por la mayoría de las instituciones encargadas de resguardar acervos documentales (TARCUS, 2005).

A partir de la normalización institucional iniciada en 1983, en el marco de la reconstrucción democrática, la UNL replanteó sus metas como institución de enseñanza superior y sus relaciones con la sociedad a través de acciones para el desarrollo de una política de recuperación y preservación de su propio pasado. Expresada en diferentes creaciones institucionales, dicha política se fue orientando a la promoción de acciones para la recuperación de diferentes aspectos de la historia de la UNL, incluyendo necesariamente la identificación, recuperación y protección de materiales, documentos, objetos y publicaciones de valor histórico-cultural y patrimonial.

En ese marco, por Resolución del Consejo Superior, en el año 2004 se creó el Archivo y Museo Histórico de la UNL, con la misión de reunir, sistematizar y albergar en condiciones apropiadas y con criterios de preservación patrimonial la documentación generada en las actividades de investigación, docencia y extensión. Con el objetivo de impulsar actividades de investigación y movilizar recursos para la recuperación y puesta en valor de su patrimonio histórico y documental mediante la generación de mecanismos, reglamentaciones y espacios específicos de trabajo, se creó el Programa Historia \& Memoria (NEIL; BERTERO, 2007).

Si bien desde sus inicios el Programa Historia \& Memoria produjo fuentes orales para satisfacer requerimientos de distintas investigaciones, fue en 2010 cuando, a partir de ese antecedente $\mathrm{y}$, dándole una reorientación a lo que se venía realizando, se resolvió producir fuentes orales con vistas a la construcción de un archivo que cubriera aspectos de la vida político-institucional de la universidad, aunque no limitado a ese solo objeto sino que también contemplara los ámbitos político, sindical y cultural de la ciudad y la provincia de Santa Fe. El punto de partida no fue pensar en posibles individuos a entrevistar sino en los ámbitos que debería cubrir el archivo. En relación con esto, se resolvió proyectar colecciones de entrevistas que pudieran interesar, en el mediano y en el largo plazo, a investigadores del campo de las ciencias sociales. 


\section{2- Consideraciones sobre la producción de fuentes orales y su archivación}

La producción de fuentes orales ya no es algo novedoso en la investigación social. Las primeras experiencias remiten al archivo de la Universidad de Columbia, organizado luego de la Segunda Guerra Mundial, con el propósito de conformar un banco de datos con testimonios de personalidades políticas. En ese momento el testimonio oral era visto como fuente de información fáctica. En la Argentina, en 1970, Luis Alberto Romero y Leandro Gutiérrez organizaron en el entonces Instituto Di Tella -hoy Universidad Torcuato Di Tella- un archivo de historia oral sobre la base de entrevistas a políticos, sindicalistas y empresarios que tuvieron actuación pública en la década de 1930 y en los años del primer peronismo (19451955).

En la segunda mitad de los años setenta, durante la última dictadura militar, algunos historiadores que debieron desarrollar sus investigaciones por fuera de las instituciones académicas oficiales -en lo que ha sido denominado "universidad de las catacumbas"- se acercaron a la historia oral. Entre ellos, cabe mencionar el trabajo pionero de Dora Schwarzstein quien, ya en democracia, organizó el Programa de Historia Oral en la Facultad de Filosofía y Letras de la Universidad de Buenos Aires, a comienzos de la década de $1990^{\mathrm{iii}}$.

Fue por esos años, precisamente en 1991, cuando el Centro Editor de América Latina publicó La historia oral, un libro compilado y prologado por Schwarzstein, que tuvo el mérito de introducir en el campo académico argentino perspectivas superadoras de los presupuestos realistas y de las aproximaciones ingenuas a la fuente oral -en el sentido de entenderla como algo transparente que restituye el pasado-. Los textos que componen el libro -escritos por Luisa Passerini, Alessandro Portelli, William Moss, Ronald Fraser, Trevor Lummis, Ronald Grele y Raphael Samuel- suponen un cuestionamiento a las limitaciones impuestas por una concepción historicista que consideraba a las fuentes orales como productos poco confiables y argumentan sobre el potencial de las mismas, al tiempo que dan cuenta de la expansión de esta práctica de investigación en Europa occidental, con una presencia notable de abordajes sociológicos, antropológicos y discursivos. En conjunto, el libro esclarece que las fuentes orales no son un simple registro de los hechos del pasado y que su valor no se agota en la posibilidad de cubrir los vacíos dejados por otro tipo de documentos. Por el contrario, se trata de productos culturales complejos que se configuran en la interrelación entre memorias públicas y privadas y entre representaciones del pasado y del presente. 
En un trabajo más reciente Portelli (2005) sostiene que la historia oral tematiza las relaciones entre narradores e investigadores, entre hechos del pasado y narraciones dialógicas del presente, lo que exige al investigador abordar tanto la dimensión fáctica como la narrativa, el referente y el significante, y considerar el espacio que media entre el pasado evocado y el presente de la enunciación. Esto se debe a que el carácter oral le imprime rasgos peculiares al testimonio y requiere considerar tanto las condiciones de producción como las mediaciones que intervienen.

El filósofo francés Paul Ricoeur (2008) señala que la pretensión de fidelidad del testimonio y la aserción de la realidad -“yo estaba allí"- hacen aflorar la opacidad de la historia personal imbricada en otras historias. Justamente preguntarnos por quién testimonia nos remite al problema de la relación entre el individuo y la sociedad. De ahí que las entrevistas no tengan que pensarse necesariamente como historias de vida o relatos biográficos (acontecimientos sucesivos asociados a un individuo). En este sentido, algunos aportes de la teoría social resultan esclarecedores al respecto.

Uno de esos aportes proviene de la perspectiva teórica de Pierre Bourdieu (1997). En esa línea, pretendemos que cada entrevista dé cuenta de la trayectoria de un agente en un campo determinado -entendido como un espacio dinámico y conflictivo, sometido a transformaciones-, de las disposiciones a actuar, de las apuestas realizadas y de las estrategias tendientes a posicionarse en el campo o en los campos en los que haya actuado. Asimismo, si tomamos como referencia la orientación desarrollada por Norbert Elias (2006), observamos que individuo y sociedad deben distinguirse, pero no separarse, pues no se trata de sustancias o de un dualismo. Justamente, su concepto de figuración alude a los sistemas de relaciones e interdependencias recíprocas, que implican diferencias de poder, tensiones y conflictos. Desde esa perspectiva, los individuos no se entienden como agentes aislados sino como parte de un entramado cambiante de relaciones.

En efecto, quien ofrece su testimonio provee informaciones y valoraciones en las que se entrelazan aspectos personales, generacionales y grupales con climas de época, formas de sociabilidad, relaciones de poder, subjetividades, prácticas y representaciones compartidas. Esto es así porque, como advierte Elizabeth Jelin (2002), si bien la capacidad de recordar y olvidar es singular, no ocurre en individuos aislados, sino inscriptos en relaciones sociales y de poder. Y como ya lo señalara Maurice Halbwachs (2011), pionero en los estudios de sociología de la memoria, incluso los recuerdos más personales se explican por los cambios 
que se producen en las relaciones de los individuos con los distintos ámbitos colectivos de pertenencia y por las transformaciones de los mismos.

Lo anterior se entrelaza con la cuestión de la memoria en el testimonio. Dado que los testimonios no restituyen el pasado sino que lo reconstruyen interpretándolo, en función de las preguntas que se plantee el investigador que los utilice es posible analizar las mediaciones entre el pasado y el presente, el peso del presente en las evaluaciones sobre el pasado, esto es, los cambios operados en el contexto político, las variaciones en las posiciones de los testigos, las presiones sociales que pueden ser reconocidas en el momento de producción de la entrevista (SCHWARZSTEIN, 2001). Es decir, todo lo que podría englobarse bajo el enunciado "contexto de emisión del testimonio".

Al decir de Jelin (2002), la memoria es una fuente crucial porque las tergiversaciones, las negaciones, los olvidos y los desplazamientos posibilitan abrir preguntas para la investigación. Todo ello, por supuesto, en función de los presupuestos teóricos y metodológicos que se plantee el investigador y en la medida que no entienda la fuente oral como una fuente inmediata de verdad ni la reduzca a algo que tiene que ser verificado con el contrapunto del documento escrito. En este sentido es que Portelli (1991) señala que el cruce entre fuentes orales y escritas no obedece al interés por verificar el testimonio, sino a la importancia de interpretarlo en su complejidad.

Pero la entrevista no sólo instituye los relatos de un sujeto, sino que también supone la presencia del entrevistador, quien está presente de distintas maneras en el testimonio en tanto que le imprime determinado carácter a las preguntas e interviene en el relato del entrevistado. La relación entre los interlocutores se establece desde el momento en que los entrevistados son seleccionados de acuerdo con determinados criterios e intereses y, casi siempre, el control del discurso permanece en manos del entrevistador. Por eso, la entrevista de investigación es una relación social que genera efectos sobre los resultados obtenidos $\mathrm{y}$, en ese sentido, se distingue de la mayoría de los intercambios corrientes (BOURDIEU, 1999).

Los planteos precedentes habilitan pensar la fuente oral como una construcción posibilitada por la relación entre un investigador y un entrevistado (seleccionado por el primero), quienes por medio de un diálogo pautado (no espontáneo) revisan, a veces fragmentariamente, aspectos significativos del pasado sobre el cual el entrevistado se presenta como testigo. En este sentido es que Leonor Arfuch $(1995,2002)$ define a la entrevista como “invención dialógica". 
Ahora bien, al decir de Ricoeur (2008), la archivación dentro de instituciones determinadas cambia el estatuto del testimonio -memoria declarada- y constituye la primera mutación historiadora de la memoria viva sometida a examen. En la medida que lo pensemos como parte de un archivo, el producto de la entrevista que es el testimonio, pasará a integrar una colección conforme criterios establecidos desde un lugar institucional. Entonces, desde el mismo momento en que se piensa la organización de un archivo oral se está operando una intervención y una selección; se establecen criterios tanto para determinar la representatividad de los testimonios como para construir una red discursiva en la que se inscriban (PÉREZ; RAITER; ZULLO, 1999). A esa cuestión nos referimos en los apartados siguientes.

\section{3- Producción, resguardo y accesibilidad}

La preservación de las fuentes orales constituye hoy un motivo de preocupación dado que, en muchos casos, su uso se restringe al que puede darle el investigador que las ha creado en el marco de una investigación particular. En este sentido, y como ha señalado Dora Schwarzstein (2002), la importancia de desarrollar colecciones temáticas en lugar de testimonios aislados y establecer criterios técnicos y prácticos para facilitar su perdurabilidad, conservación, clasificación y accesibilidad es una tarea imprescindible en el marco de un campo que en las últimas décadas ha adquirido una notable expansión.

Desde los comienzos de la organización del archivo hemos desarrollado una forma de trabajo que sintetizamos a continuación. En primer lugar, tomamos contacto con el potencial entrevistado, explicitamos los propósitos y acordamos los tópicos de la conversación. En función de los desafíos que se nos plantean, en algunos casos solicitamos la colaboración de colegas cuyas investigaciones tengan afinidad con el tema de la entrevista. Una vez que el entrevistado accede, la entrevista se filma en formato Full HD. El original se guarda y se hace una copia para la consulta. Se firma un documento con el entrevistado, en virtud del cual cede su testimonio al archivo para la consulta pública.

Dado que los testimonios pueden contener informaciones sensibles, se determinan criterios para proteger la privacidad y, en caso que el entrevistado lo solicite, se establecen límites de para la consulta. Se firma un protocolo entre el archivo y cada entrevistado, en el que constan las pautas (acceso sin restricciones, fijación de un período de guarda antes de ponerlo a la consulta pública, entre otros). El documento original queda en poder del archivo 
y al entrevistado se le entrega una copia. Por su parte, quien realice una consulta debe completar un protocolo de uso en el que constan sus datos personales, la institución de pertenencia y los fines de la consulta.

A partir de cada entrevista se generan tres fichas. Dos de ellas acompañan a la entrevista en el archivo. En una están consignados los datos personales del entrevistado y aspectos relevantes de su trayectoria. Una segunda ficha contiene la fecha de realización de la entrevista, el período que abarca, el tema, las personas mencionadas y los descriptores. Sobre la base de estas dos se elabora una tercera ficha que figura en la web. Contiene la siguiente información: fecha de realización de la entrevista, período que abarca, tema, duración, palabras clave y resumen biográfico. Estas fichas pueden consultarse en: https://www.unl.edu.ar/extension/categorias/programas/historia-memoria/. Las entrevistas completas pueden consultarse en la Biblioteca Centralizada FHUC-FADU-ISM de la Ciudad Universitaria, en la ciudad de Santa Fe.

\section{4- Las colecciones del archivo de historia oral}

El archivo se organiza sobre la base de áreas temáticas a partir de las cuales se conforman colecciones y series de fuentes orales. Se entiende por serie un conjunto de entrevistas más o menos homogéneas (en función del tema, período, etc.). Un conjunto de series conforma una colección. Por ejemplo, la colección de fuentes sobre la UNL está integrada por distintas series; lo mismo cabe decir de la colección sobre el campo político. Las entrevistas se realizan a partir de un cuestionario semiestructurado, de modo que los testimonios sobre una misma temática tengan un formato similar. A continuación, se explicita la conformación de las colecciones.

\section{1- Colección Universidad Nacional del Litoral}

Para organizar esta colección identificamos cuatro segmentos temporales, a saber, los años sesenta, entre la caída del peronismo y el golpe de Onganía en 1966; los años setenta, desde ese acontecimiento hasta el cierre autoritario de 1976 (SUASNÁBAR, 2004); los años de la última dictadura militar (1976-1983) y la etapa de la normalización en las universidades 
nacionales, desde la reconstrucción democrática iniciada a fines de 1983. La colección está constituida por tres series, que se detallan a continuación.

\section{a- Serie Gobierno de la Universidad}

Contiene entrevistas a rectores y otros funcionarios de la UNL. Las entrevistas exploran la relación particular de la UNL con el contexto político nacional, las orientaciones de la política académica y las tensiones al interior de la institución.

\section{b-Serie Movimiento Estudiantil}

Esta serie busca reconstruir una red discursiva que dé cuenta de las distintas orientaciones de la política estudiantil universitaria desde mediados de los años sesenta, haciendo foco en la trayectoria del reformismo y en sus variadas experiencias, ligadas al radicalismo y a la izquierda. El propósito consiste en conformar un corpus representativo de la pluralidad de memorias.

\section{c- Serie Instituto de Cinematografía}

La serie está dedicada a la producción de testimonios sobre dicho instituto, creado en 1956 bajo el impulso de Fernando Birriv y de Ángela Romera Vera, directora del Instituto Social de la UNL. Desde la creación hasta su cierre definitivo en 1976, el Instituto desarrolló una tarea formativa, en lo que a documentalismo se refiere, y acciones de extensión a la comunidad, y estuvo atravesado por el debate estético-político que se desarrolló entre fines de los años sesenta y principios de los setenta (SARLO, 1998). Las entrevistas que componen esta serie se centran en el derrotero del Instituto de Cinematografía y dan cuenta de las relaciones cambiantes y no exentas de conflicto entre la universidad y el contexto políticointelectual de los años sesenta y de la primera mitad de los setenta (NEIL; PERALTA; PRIAMO; BECEYRO, 2007).

Dentro de esta colección está previsto incorporar una serie sobre grupos académicos, orientada a indagar los rasgos y características del clima intelectual universitario atravesado por las orientaciones de la políticas académicas, la formación de grupos ligados a la institucionalización de disciplinas y a la creación de institutos de investigación; y otra serie abocada a indagar la formación de grupos afines a la política universitaria durante los períodos autoritarios, en particular durante la última dictadura militar. 


\section{2- Colección Campo Político}

La construcción de esta colección responde a la inquietud de considerar los procesos provinciales como espacios de producción de lo político que, con sus propios ritmos y especificidades, contribuyen a configurar los procesos nacionales (FERRARRI; CLOSA, 2015). De ahí que la colección busca explorar los variados aspectos del campo político provincial a partir de entrevistas a protagonistas del sistema político de la segunda mitad del siglo XX y a quienes hayan desarrollado acciones en la esfera pública vinculadas a los derechos humanos durante la última dictadura militar. Asimismo, dadas las particulares características del ámbito local, una serie está dedicada a las relaciones entre política y religión a partir de la segunda mitad del siglo XX. La colección está compuesta por cuatro series.

\section{a- Serie Partidos y Gobierno}

Como es sabido, la reconstrucción democrática iniciada en 1983 habilita la oportunidad de edificar un poder legítimo y estable. Bipartidismo y alternancia política son características de esta etapa. A partir de 1989, tras el telón de fondo de la democracia se instalará una práctica de gobierno ubicada en una zona ambigua, entre los imperativos de la política y la vigencia del Estado de derecho (QUIROGA, 2006).

Si bien se fija como límite temporal 1983, ello no es obstáculo para tratar también la etapa previa ya que muchos testigos iniciaron sus trayectorias políticas a comienzos de los años cincuenta. En primer término, se entrevistó a políticos de los dos partidos mayoritarios (Unión Cívica Radical y Partido Justicialista) para indagar sus vínculos a nivel local y nacional, las estrategias (por dentro y por fuera de cada partido) a nivel local, sus trayectorias durante la última dictadura militar y la formación de la Multipartidaria ${ }^{\mathrm{v}}$. En lo que respecta a la Unión Cívica Radical, se indaga la inserción de dirigentes santafesinos en el Movimiento de Renovación y Cambio y en la Junta Coordinadora Nacional. En lo que respecta al Partido Justicialista, el derrotero de la renovación peronista en la década de 1980 y la emergencia del menemismo en la década siguiente.

Tras haber realizado entrevistas a miembros de los dos partidos mayoritarios, se avanzó en la producción de testimonios de dirigentes del Partido Demócrata Progresista 
(PDP), del Movimiento de Integración y Desarrollo (MID), del Partido Socialista (PS) y del Partido Comunista (PC). La serie incluye entrevistas a la mayoría de los gobernadores que se sucedieron desde 1983, a ex ministros y a legisladores nacionales y provinciales. Asimismo, a militantes de base con el propósito de explorar las relaciones construidas entre éstos y los dirigentes partidarios, la militancia barrial y las modalidades de reclutamiento de militantes en los distintos partidos.

\section{b- Serie Constitución del peronismo en la ciudad de Santa Fe (1943-1946)}

En su etapa formativa, el peronismo santafesino se conformó a partir de las tradiciones nacionalista, radical y católica (MACOR, 2003). Constituida por veintiocho entrevistas producidas en el marco de una investigación realizada en la UNL en la segunda mitad de la década del noventa, esta serie contiene testimonios de intelectuales, funcionarios provinciales, estudiantes universitarios, profesionales y dirigentes sindicales que formaron parte del proceso de formación del peronismo en la ciudad de Santa Fe. Las entrevistas recogen testimonios de quienes adhirieron al nuevo movimiento político y de quienes se constituyeron como opositores al mismo.

\section{c- Serie Política y Religión}

Dado que el Movimiento de Sacerdotes para el Tercer Mundo (1967-1976) organización nacida en el universo católico transformado por la renovación posconciliar (TOURIS, 2005)- tuvo un desarrollo considerable en la ciudad de Santa Fe, se conformó una serie sobre el rol jugado por los católicos en el proceso de radicalización política. A partir de los testimonios de sacerdotes y laicos es posible reconstruir la red de relaciones y prácticas. Las entrevistas atienden especialmente a los vínculos entre diversas instituciones de la iglesia católica, a la circulación de ideas y a la convergencia de laicos y sacerdotes en espacios de sociabilidad diversos como el seminario, las Universidad Católica de Santa Fe, los colegios, las parroquias y los barrios populares. Otras dimensiones que se tienen en cuenta son las relaciones entre catolicismo, peronismo y marxismo y la opción por la lucha armada.

\section{d-Serie Derechos Humanos}

A partir de los años ochenta se ha venido configurando un nuevo campo de análisis en las ciencias sociales latinoamericanas, centrado en los derechos humanos y en las memorias 
de la represión y de la violencia política (JELIN, 2004). El trabajo con testimonios orales es una de las vías para el estudio de la conformación de las memorias sociales, tanto a nivel nacional como en los ámbitos locales en los que el movimiento de derechos humanos tuvo desarrollo. En la provincia de Santa Fe, en las ciudades de Santa Fe y Rosario, el movimiento de derechos humanos comenzó a configurarse durante los primeros años de la última dictadura militar. El acuerdo básico en torno al vínculo entre justicia, democracia y derechos humanos fue fundamental para sentar las bases simbólicas, éticas y políticas de la reconstrucción democrática en 1983.

La serie se organiza a partir de los testimonios de quienes fueron fundadores y referentes de las principales organizaciones de la ciudad de Santa Fe: Familiares de Detenidos Desaparecidos por Razones Políticas (1977), Asamblea Permanente por los Derechos Humanos (1980/1981), Movimiento Ecuménico por los Derechos Humanos (1980/1981) y Madres de Plaza de Mayo (en una primera etapa sus referentes estuvieron integradas a Familiares, por lo que la agrupación se constituyó definitivamente en 1987). Ya en la etapa constitucional iniciada a fines de 1983, la Comisión Nacional sobre la Desaparición de Personas (CONADEP) llevó a cabo la tarea de recolección de testimonios de las víctimas de la represión ilegal durante la última dictadura militar. En la provincia de Santa Fe funcionaron la Delegación Zona Sur, con sede en la ciudad de Rosario, y la Subdelegación Zona Norte, con sede en la ciudad de Santa Fe.

\section{3- Colección Campo Cultural}

Dada la amplitud de lo que suele entenderse por cultura, nos basamos en Oscar Terán (1991), para quien los años que median entre 1956 y 1966 son los de la constitución de una serie de núcleos ideológicos en el campo cultural argentino, altamente sensibles a los acontecimientos políticos. En referencia a los cambios en la vida cultural de la ciudad de Buenos Aires durante la década del sesenta, Luis Alberto Romero (1994) identifica las vanguardias artísticas, que tendrán su centro de expresión en el Instituto Di Tella, y la universidad como el principal foco de la renovación, luego de la caída del peronismo. Para esos mismos años, Beatriz Sarlo (1998) señala los conflictos entre vanguardistas, por un lado, y partidarios del realismo y del arte comprometido, por el otro. Para la constitución de una colección sobre esta temática, ajustada al espacio local, se planteó la necesidad de producir 
fuentes orales a partir de las cuales pudiera reconstruirse un mapa de la actividad cultural en la ciudad de Santa Fe para contribuir, de este modo, al desarrollo de investigaciones sobre el campo cultural en la segunda mitad del siglo XX.

Siguiendo los planteos críticos de Raymond Williams (1994) en relación con las investigaciones en sociología de la cultura, las entrevistas no se piensan desde una visión sustancialista del mundo cultural -como la idea del "artista y su público" o la "biografía del artista o el intelectual"-, sino que apuntan a que el diálogo entre entrevistador y entrevistado pueda dar cuenta de lo siguiente: prácticas culturales, instituciones a través de las cuales se desarrollaba la producción cultural (el lugar que le cupo a la UNL), formaciones culturales (los modos en que los productores culturales fueron organizados o se organizaron a sí mismos), las relaciones de los productores con el mercado cultural y con los entes gubernamentales, las formas de circulación de los productores y las obras, las relaciones con otros centros de producción cultural (Buenos Aires, Rosario, Paraná, Córdoba). Se conforma así una colección integrada por testimonios que provienen de distintos subcampos de la cultura: periodismo, edición, teatro, cine, música, crítica cultural.

\section{4- Colección Poder Judicial}

Esta colección tiene por objeto de la realización de entrevistas a funcionarios del Poder Judicial de la provincia de Santa Fe, fiscales, jueces y ministros de la Corte Suprema de Justicia provincial.

\section{5- Colección Economía y Sociedad}

Esta colección se aboca a la producción de fuentes orales sobre organizaciones corporativas. Está integrada por dos series -Organizaciones Empresariales y Organizaciones Sindicales- de las que hasta el momento sólo se pudo avanzar en la constitución de la segunda, que contiene entrevistas a dirigentes del magisterio, de sindicatos de trabajadores estatales y del sindicato de la Sanidad, todos ellos de la ciudad de Santa Fe, y de la Unión Obrera Metalúrgica (UOM) de la ciudad de Villa Constitución. 


\section{5- Consideraciones finales}

Los archivos orales constituyen repositorios con potencialidad analítica para el estudio del pasado reciente $\mathrm{y}$, en lo que respecta a la construcción de las memorias, permiten esclarecer las operaciones de selectividad y las interpretaciones cambiantes acerca del pasado. El proceso de construcción de un archivo oral no tiene un punto de llegada. Las colecciones y las series que las constituyen están en permanente conformación (y revisión) a medida que se van acrecentando. Además, si bien una entrevista forma parte de una serie determinada, puede tener conexiones e intersecciones con una entrevista de otra serie (por caso, entre las de política y sindicalismo y entre las de universidad y cultura). Esta característica obedece tanto al contenido mismo de las entrevistas como al uso que de las mismas hacen los investigadores, en función de sus perspectivas teórico-metodológicas.

Como sabemos, el documento histórico siempre está abierto a la interpretación, lo que vale también para el testimonio oral. Tal vez uno de los aspectos más desafiantes e interesantes de la producción de fuentes orales radica en que se pone de manifiesto que el pasado no es una entidad objetiva ni consensual sino un ámbito de tensiones y disputas sobre su sentido (JELIN, 2002). La trama de relaciones, desde el presente hacia el pasado, queda como un problema abierto hacia el porvenir porque, como sostiene Arlette Farge (1991), la historia -en el sentido de conocimiento- nunca es repetición del archivo, sino desinstalación con respecto a él.

Podemos saber lo que los testimonios significan hoy para nosotros, pero no podemos anticipar lo que significarán en el futuro. Justamente, ese carácter abierto e inestable del testimonio nos impone la necesidad de preservar esa producción y garantizar las mejores condiciones para que en el futuro llegue a otros destinatarios que no seremos nosotros.

\section{6- Referencias}

ARFUCH, L. La entrevista, una invención dialógica. Buenos Aires: Paidós, 1995. 161p.

ARFUCH, L. El espacio biográfico, dilemas de la subjetividad contemporánea. Buenos Aires: Fondo de Cultura Económica, 2002. 272p.

BOURDIEU, P. La ilusión biográfica. En: . Razones prácticas. Sobre la teoría de la acción. Barcelona: Anagrama, 1997. Cap. 3. Anexo I, p.74-83. 
BOURDIEU, P. (Dirección). La miseria del mundo. Buenos Aires: Fondo de Cultura Económica, 1999. 564p.

ELIAS, N. Sociología fundamental. Barcelona: Gedisa, 2006. 216p.

FARGE, A. La atracción del archivo. Valencia: Edicions Alfons El Magnànim, 1991. 97p.

FERRARI, M.; CLOSA, G. Los partidos políticos mayoritarios durante la reconstrucción democrática. Córdoba y Buenos Aires, 1982-1991. En: FERRARI, M.; GORDILLO, M. (compiladoras). La reconstrucción democrática en clave provincial. Rosario: Prohistoria, 2015. Cap. 1, p.29-64.

HALBWACHS, M. La memoria colectiva. Buenos Aires: Miño y Dávila, 2011. 251p.

JELIN, E. Los trabajos de la memoria. Madrid y Buenos Aires: Siglo XXI, 2002. 146p.

JELIN, E. Los derechos humanos y la memoria de la violencia política: la construcción de un campo nuevo en las ciencias sociales. Estudios Sociales. Revista Universitaria Semestral, Universidad Nacional del Litoral, Santa Fe, n. 27, p. 91-113, 2004.

MACOR, D. Las tradiciones políticas en los orígenes del peronismo santafesino. En: MACOR, D.; TCACH, C. (editores). La invención del peronismo en el interior del país. Santa Fe: Ediciones UNL, 2003. p. 85-110.

NEIL, C.; PERALTA, S.; PRIAMO, L.; BECEYRO, R. Fotogramas santafesinos. Instituto de Cinematografía de la UNL, 1956/1976. Santa Fe: Ediciones UNL, 2007. 241p.

NEIL, C; BERTERO, E. Propuestas para la construcción de un pasado colectivo. Revista ConCiencia, Universidad Nacional del Litoral, Santa Fe, n. 18, p.21-22, 2007.

PÉREZ, S.; RAITER, A.; ZULLO, J. Hacer historia con herramientas textuales. En: RAITER, A. y otros. Discurso y ciencia social. Buenos Aires: EUDEBA, 1999. Cap. 3, p.51-61.

PORTELLI, A. Lo que hace diferente a la historia oral. En: AA.VV. La historia oral. Buenos Aires: Centro Editor de América Latina, 1991. Cap. 2, p.36-52.

PORTELLI, A. El uso de la entrevista en la historia oral. Historia, memoria y pasado reciente. Anuario $\mathrm{N}^{\circ} \mathbf{2 0}, \mathbf{2 0 0 3} / \mathbf{2 0 0 4}$, Escuela de Historia, Universidad Nacional de Rosario y Homo Sapiens, 2005. p. 35-48.

QUIROGA, H. La política en tiempos de dictadura y democracia. En: QUIROGA, H; $\mathrm{TCACH}, \mathrm{C}$. Argentina 1976-2006. Entre la sombra de la dictadura y el futuro de la democracia. Rosario: Homo Sapiens Ediciones y Universidad Nacional del Litoral, 2006. Cap. 4, p.69-96.

RICOEUR, P. La historia, la memoria, el olvido. Buenos Aires: Fondo de Cultura Económica, 2008. 673p. 
ROMERO, L. A. Breve historia contemporánea de la Argentina. Buenos Aires: Fondo de Cultura Económica, 1994. 414p.

SARLO, B. La máquina cultural. Maestras, traductores y vanguardistas. Buenos Aires: Ariel, 1998. 292p.

SCHWARZSTEIN, D. Entre Franco y Perón. Memoria e identidad del exilio republicano español en la Argentina. Barcelona: Crítica, 2001. 293p.

SCHWARZSTEIN, D. El lugar de las fuentes orales en los archivos: una cuestión a debate. Estudios Sociales. Revista Universitaria Semestral, Universidad Nacional del Litoral, Santa Fe, n. 22-23, p.11-22, 2002.

SUASNÁBAR, C. Universidad e intelectuales. Educación y política en la Argentina (1955-1976). Buenos Aires: FLACSO-Manantial, 2004. 301p.

TARCUS, H. ¿El drenaje patrimonial como destino? Bibliotecas, hemerotecas y archivos argentinos, un caso de desarrollo cultural. Revista La Biblioteca, Biblioteca Nacional, Buenos Aires, n. 1, p.22-29, 2004-2005.

TERÁN, O. Nuestros años sesentas. Buenos Aires: Puntosur, 1991. 193p.

TOURIS, C. Neo-integralismo, denuncia profética y Revolución en la trayectoria del Movimiento de Sacerdotes para el Tercer Mundo. Prismas. Revista de historia intelectual, Universidad Nacional de Quilmes, n. 9, p. 229-239, 2005.

WILLIAMS, R. Sociología de la cultura. Buenos Aires: Paidós, 1994. 231p.

\section{Sobre las autoras}

Fabiana Alonso. Profesora en Historia y Magister en Ciencias Sociales por la Universidad Nacional del Litoral (UNL), Argentina. Profesora Adjunta de Teoría de la Historia en la Facultad de Humanidades y Ciencias de la UNL. Profesora Asociada en la Licenciatura en Historia de la Universidad Autónoma de Entre Ríos (UADER). Investigadora categorizada en la UNL. Directora del Programa Historia \& Memoria de la Secretaría de Extensión de la UNL.

Eliana Bertero. Profesora en Historia graduada en la Universidad Nacional del Litoral (UNL), Argentina. Investiga sobre historia de la universidad y es auxiliar docente en la cátedra Historia Institucional Argentina de la Facultad de Ciencias Jurídicas y Sociales de la UNL. 
Docente del Instituto Superior de Profesorado No 8 "Alte. G. Brown" de Santa Fe. Coordinadora del Programa Historia \& Memoria de la Secretaría de Extensión de la UNL.

\section{Notas}

i La Reforma Universitaria acaecida en la Universidad de Córdoba en 1918 fue parte del proceso de modernización y democratización de la sociedad argentina. Al interior de la universidad significó una transformación institucional y académica que modificó las estructuras de gobierno y las instancias de selección de los cuerpos docentes. Se constituyó también en un movimiento cultural con proyección en América Latina.

ii Memoria del año 1927. El documento, publicado bajo el sello de la UNL, describe minuciosamente los documentos que registran la vida institucional y cultural de la institución creada en 1919.

iii Otras iniciativas más recientes en la Argentina son los archivos dedicados a las memorias de la represión, como Memoria Abierta, archivo oral creado en 2001 por un grupo de organismos de derechos humanos. En la misma línea, el archivo oral sobre memorias de la represión en Córdoba, que es parte del Archivo Provincial de la Memoria de Córdoba. En el marco del Programa de Historia Política del Instituto Gino Germani de la Universidad de Buenos Aires se organizó un archivo oral en 2003, dedicado a la producción de testimonios de dirigentes políticos, sindicales, economistas, militares, académicos, dirigentes de organizaciones de la sociedad civil y de organizaciones armadas.

${ }^{\text {iv }}$ Entre sus películas más conocidas pueden citarse Tire dié (1958) y Los inundados (1961), realizadas ambas en el marco del Instituto de Cinematografía.

${ }^{\vee}$ Se conoce con el nombre de Multipartidaria a la organización que, en 1981, durante la última dictadura militar argentina, fue creada por la Unión Cívica Radical, los partidos Justicialista, Intransigente y Demócrata Cristiano y el Movimiento de Integración y Desarrollo. 